\title{
Pharmacology, Part 5: CT and MRI Contrast Media
}

\author{
Geoffrey M. Currie \\ Faculty of Science, Charles Sturt University, Wagga Wagga, Australia, and Regis University, Boston, Massachusetts
}

CE credit: For CE credit, you can access the test for this article, as well as additional JNMT CE tests, online at https://www.snmmilearningcenter.org. Complete the test online no later than September 2022. Your online test will be scored immediately. You may make 3 attempts to pass the test and must answer $80 \%$ of the questions correctly to receive $1.0 \mathrm{CEH}$ (Continuing Education Hour) credit. SNMMI members will have their CEH credit added to their VOICE transcript automatically; nonmembers will be able to print out a CE certificate upon successfully completing the test. The online test is free to SNMMI members; nonmembers must pay $\$ 15.00$ by credit card when logging onto the website to take the test.

Pharmacology principles provide key understanding that underpins the clinical and research roles of nuclear medicine practitioners. The scope of practice of the nuclear medicine technologist demands knowledge and understanding of indications, contraindications, warnings, precautions, proper use, drug interactions, and adverse reactions for each medication to be used. This article is part of a series that aims to enhance understanding of pharmacologic principles relevant to nuclear medicine. This article will build on the introductory concepts, terminology, and principles of pharmacology explored in the first 2 articles in the series. Specifically, this article will focus on the pharmacologic principles and adverse reactions associated with iodinated and gadolinium contrast media used in $\mathrm{CT}$ and $\mathrm{MRI}$, respectively.

Key Words: PET/CT; PET/MRI; SPECT/CT; gadolinium; iodinated contrast; pharmacology

J Nucl Med Technol 2019; 47:189-202

DOI: 10.2967/jnmt.118.220012

I imaging, a contrast agent is any agent that is administered to the patient in order to improve visualization of an organ, tissue, or pathologic condition. Generally, contrast agents are positive (increase opacity), but some negative contrast agents (decreasing opacity) exist (e.g., air or gases). In the context of this article, the positive contrast agents associated with iodine and gadolinium will be discussed. Specifically, iodinated contrast agents will be outlined with reference to CT, and gadolinium for MRI, as appropriate for the target readership. It should, however, be noted that both gadolinium and iodinated contrast agents are also used outside MRI and CT, respectively.

Although contrast agents have become safer and better tolerated in recent decades, adverse reactions still occur to

\footnotetext{
Received Sep. 4, 2018; revision accepted Dec. 20, 2018.

For correspondence or reprints contact: Geoffrey M. Currie, Faculty of Science, Locked Bag 588, Charles Sturt University, Wagga Wagga 2678, Australia.

E-mail: gcurrie@csu.edu.au

Published online Feb. 15, 2019.

COPYRIGHT (C) 2019 by the Society of Nuclear Medicine and Molecular Imaging.
}

varying degrees. Consequently, it is essential that those administering contrast agents or monitoring patients after contrast administration be familiar with the associated pharmacology and adverse reactions. The foundations of pharmacology and pharmacokinetics outlined in previous articles in this series, including specific medication profiles, will be considered assumed knowledge (1-5). This insight and understanding will facilitate early detection of adverse reactions and inform a response with the most appropriate management.

\section{CT CONTRAST MEDIA}

\section{Introduction}

Ideally, a contrast agent for CT would be able to provide opacification of blood vessels, organs, and tissues without altering physiology or producing toxicity (6). The iodine molecule can absorb x-rays to provide contrast and has been the basis of intravenous contrast since 1929 (6). The development of organic iodinated compounds emerged because the inorganic sodium iodide was too toxic for regular or routine use (6). Today, CT contrast agents are based on the structure that emerged in the 1950s: triiodinated derivatives of benzoic acid that were ionic and of high osmolality (6-9). Current agents are either monomers or dimers (1 or 2 benzene rings), ionic or nonionic, high- or low-osmolality or isotonic, and of varying viscosity (7-10). Almost all current CT contrast agents are iodine-based. The emergence of nonionic CT contrast agents created the misnomer that they were noniodinated; nonionic does not mean no iodine. More than 50 million CT studies are performed in the United States each year, of which an estimated 50\% include intravenous contrast media (11). Worldwide, there are an estimated 75 million intravenous CT contrast doses administered annually (10).

\section{Properties}

Iodinated contrast agents have several key properties that influence their behavior, efficiency, and adverse reaction profile (Table 1). Interplay among these properties optimizes the degree of radioopacification and tolerability. One of these properties, iodine concentration, is the amount of iodine (mg) per unit volume $(\mathrm{mL})$ of the contrast solution administered. 
TABLE 1

Key Properties of lodinated Contrast Agents That Influence Their Behavior, Efficiency, and Adverse Reaction Risk

\begin{tabular}{|c|c|c|c|}
\hline Contrast agent & lodine concentration $(\mathrm{mg} / \mathrm{mL})$ & Osmolality (mOsm $/ \mathrm{kg}$ water) & Viscosity $(\mathrm{mPa} / \mathrm{s})\left(37^{\circ} \mathrm{C}\right)$ \\
\hline Ionic monomer (HOCM) & Up to 400 & $1,400-2,100$ & \\
\hline Ionic dimer (HOCM) & 320 & 600 & \\
\hline Nonionic monomer (LOCM) & Up to 350 & $600-800$ & \\
\hline Nonionic dimer (IOCM) & 320 & 290 & \\
\hline Human serum & $3.2-4$ & 290 & $1.5-2.0$ \\
\hline \multicolumn{4}{|l|}{ Ionic monomers } \\
\hline Amidotrizoic acid & 146 & 1,690 & 8.5 \\
\hline Amidotrizoate-meg & 350 & 1,530 & 7.5 \\
\hline loxitalaminic acid & & 2,130 & \\
\hline \multicolumn{4}{|l|}{ Nonionic monomers } \\
\hline lohexol & $240,300,350$ & $500,690,880$ & $3.3,6.1,10.6$ \\
\hline lopamidol & $200,300,370$ & $413,616,796$ & $2.0,4.7,8.6$ \\
\hline Ioxilan & 350 & 695 & 4.6 \\
\hline lopromide & 370 & 780 & 9.5 \\
\hline loversol & 320 & 702 & 5.8 \\
\hline lomeprol & 350 & 618 & 7.5 \\
\hline lobitridol & 350 & 915 & 10.0 \\
\hline Ionic dimers ioxaglate & 320 & 580 & 7.5 \\
\hline \multicolumn{4}{|l|}{ Nonionic dimers } \\
\hline lodixanol & 320 & 290 & 11.4 \\
\hline lotrolan & 300 & 320 & 8.1 \\
\hline
\end{tabular}

Optimizing key properties of intravenous CT contrast agents has resulted in evolution to those that are easier to use, with lower intravenous toxicity and fewer adverse effects (frequency and severity) $(6,8,12)$.

Another, osmolality, is a measure of the number of active particles when the agent is dissolved in $1 \mathrm{~kg}$ of water $(\mathrm{mOsm} / \mathrm{kg})(12,13)$. Other properties include viscosity, the internal or flow friction, resistance, or thickness of the fluid $(8,12)$; ionicity, the tendency for the agent to separate into charged species (ions) when dissolved in solution; oligomerization, the chemical structure (either monomer or dimer); and dose delivery, which can vary in both relative $(\mathrm{mg} / \mathrm{kg})$ and absolute (mg iodine) terms and in time course.

Iodine concentration among iodinated CT contrast agents ranges from $11 \%$ to $46 \%$, with higher iodine concentrations providing both better radioopacification and a higher risk of adverse reactions (12). Today, most current-generation CT contrast agents are nonionic; however, they often vary significantly in terms of iodine content $(7,12)$. The dimer configuration is designed to reduce the risk of adverse reactions and toxicity without compromising radioopacification. Typically, the iodine concentration of common iodinated contrast agents is on the order of $300 \mathrm{mg} / \mathrm{mL}$; however, the range is from 200 to $400 \mathrm{mg} / \mathrm{mL}$ (Table 1). Indeed, the same contrast agent maybe be marketed and branded with varying iodine concentrations (e.g., Omnipaque 240, Omnipaque 300, and Omnipaque 370; GE Healthcare). Increasing iodine concentration increases viscosity.

Osmolality introduces the concept of tonicity-hypertonic or isotonic, for example. Tonicity relates to the impact of the osmolality of a solution on the surrounding cells (12). Isotonic solutions (like most radiopharmaceuticals) have an osmolality that equals that of blood $(290 \mathrm{mOsm} / \mathrm{kg}$ of water) and thus has no impact on surrounding cells (12).
Hypertonic solutions have a higher osmolality than blood, resulting in water being drawn out of blood cells (12). Iodinated contrast agents in CT can have an osmolality 7 times that of blood. Hypotonic solutions have a lower osmolality than blood, resulting in water being taken into blood cells (12). Iodinated contrast agents are classified as high-osmolality contrast media (HOCM), low-osmolality contrast media (LOCM), or isoosmolar contrast media (IOCM) (Table 1) (12). There are no hypotonic iodinated contrast agents. Both HOCM and LOCM are hypertonic, just to varying degrees. Older iodinated contrast agents are typically $\mathrm{HOCM}(1,300-2,140 \mathrm{mOsm} / \mathrm{kg}$ of water $)$, whereas newer contrast agents developed from the 1980s are typically LOCM (600-850 mOsm $/ \mathrm{kg}$ of water). Osmolality contributes to the incidence of nonanaphylactoid adverse reactions mediated by endothelial damage, movement of fluid among compartments, and cell deformation. IOCM iodinated contrast agents are a more recent development, with osmolality that equals that of blood. Given that the aim of iodinated CT contrast agents is to have a sufficient iodine concentration for radioopacification, the ratio of iodine atoms to particles in solution is important; HOCM have a ratio of 0.5 ; LOCM, 3.0; and IOCM, 6.0. Adverse effects attributable to osmolality include pain, flushing, nausea, vomiting, and dehydration (12). Contrast osmolality higher than blood (HOCM and LOCM) results in movement of water from interstitial spaces into the vascular compartment, which causes increased blood viscosity, endothelial damage, hypervolemia, vasodilation, edema with neurotoxicity, decreased myocardial contractility, and toxicity (6). 
The viscosity of iodinated CT contrast agents is determined by their flow friction, resistance, or thickness $(8,12)$. Iodinated contrast agents are significantly more viscous than radiopharmaceuticals, and this attribute has an impact on intravenous injectability, flow rate, and gauge of canula required. Viscosity can be reduced by injecting the agent at body temperature $\left(37^{\circ} \mathrm{C}\right)$ rather than room temperature (the viscosity of an iodinated contrast agent at $20^{\circ} \mathrm{C}$ is approximately twice that of the same agent at $37^{\circ} \mathrm{C}(12)$ ). Viscosity is influenced by the molecular structure and composition of the contrast agent, with inclusion of sodium decreasing viscosity but increasing endothelial irritation compared with meglumine. LOCM has higher viscosity than HOCM, and viscosity increases with iodine content (Table 1) (12). Viscosity plays an important role in renal tolerance, contributing significantly to the risk of contrast-induced nephrotoxicity (7). High-viscosity intravenous iodinated contrast agents require longer infusion times $(8)$, whereas low viscosity allows the use of a rapid bolus (12) - a key factor in optimizing the contrast agent for imaging protocols. Highviscosity intravenous contrast agents also influence the local circulation (8). Water is used as the reference standard for viscosity at $1 \mathrm{cP}$, plasma is then $1.5-2 \mathrm{cP}$, and iodinated CT contrast agents range from $2.0 \mathrm{cP}$ for lowiodine-concentration, nonionic, monomer-based contrast agents up to $11.4 \mathrm{cP}(12)$.

Contrast agents used intravenously in $\mathrm{CT}$ are triiodinated derivatives of benzoic acid (Fig. 1) (6-9). A monomer is a simple molecule or base unit that can undergo polymerization. In the case of iodinated contrast agents, the monomer is a 2,4,6-triiodinated benzene ring (Fig. 1). A dimer is 2 monomer units bonded after polymerization to form a

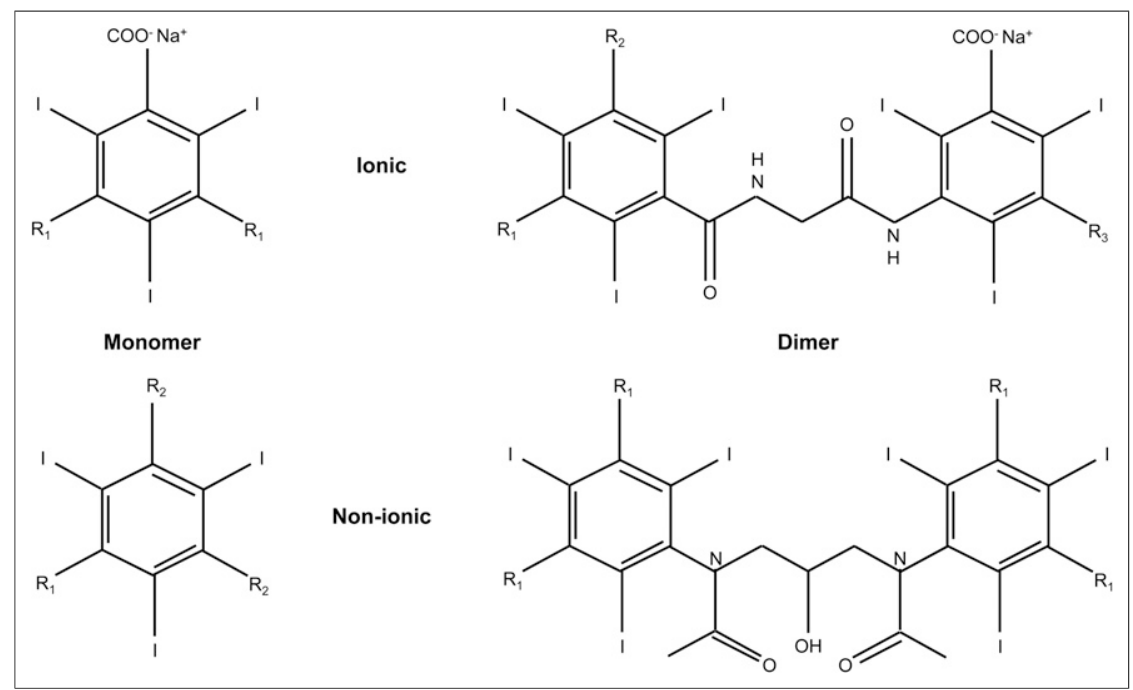

FIGURE 1. Chemical structure of iodinated CT contrast agents is based on 2,4,6triiodinated benzene ring and provides 4 major classifications of iodinated CT contrast agents: ionic monomer, ionic dimer, nonionic monomer, and nonionic dimer. For ionic contrast media, carboxyl group $(\mathrm{COOH})$ ionizes $\left(\mathrm{COO}^{-}\right)$with sodium or meglumine to form anion and cation pairs. Side chains $(R)$ vary but tend to be longer for nonionic contrast media. 2-unit oligomer. Monomers have higher osmolality than dimers, with dimers typically being LOCM or IOCM (Table 1) (7). Covalently bonded iodine atom associated with $C T$ contrast agents are within the range of $\mathrm{x}$-ray wavelengths so can produce attenuation (10). This is enhanced by the close proximity of the triiodinated configuration, enhancing attenuation properties (10). Importantly, the benzene ring structure reduces the risk of toxicity $(10)$.

Ionic iodinated CT contrast agents dissociate into ion pairs whereas nonionic do not (Fig. 1) (13). Nonionic does not mean noniodinated, nor does it mean LOCM even though most nonionic contrast is LOCM. Fully saturated triiodinated benzoic acid derivative monomer CT contrast agents dissociate into ions in solution, with the anion containing the iodine atoms and the cation containing sodium or meglumine $(6,8,9,12,13)$. These are typically hypertonic (osmolality 5 times that of blood) and alter the plasma tonicity (6). Consequently, adverse reactions are common $(6,12)$. The dimer form (2 triiodinated benzoic acid rings) allows high iodine content but low osmolality because a single cation is still all that is required $(6,8,9,12)$. Only the anion is radioopaque, because it carries the iodine atoms, but the cation is needed for the solution to be formed ( 1 ).

Nonionic monomer CT contrast agents have a longer side chain than ionic monomers (Fig. 1), and this increases the molecular weight, decreases the osmolality, but does not change the iodine concentration $(6,8,12)$. The nonionic dimer structures tend to be isotonic (6). Low-osmolality agents are less toxic and have fewer adverse reactions (6). The 4 major classifications of iodinated CT contrast agents include ionic monomer, ionic dimer, nonionic monomer, and nonionic dimer (Fig. 1) (8-10).

Individual doses for patients should be tailored in consideration of the properties outlined above, as well as in consideration of iodine concentration; volume of dose; patient height, weight, age, and sex; venous accessibility; renal function; pharmacokinetic model; injection rate; target organ; target enhancement; and CT scanner and imaging protocol (14).

\section{Mechanism of Action}

Although a discussion of the physical principles of $\mathrm{x}$-ray production in $\mathrm{CT}$ is beyond the scope of this article, it is important to briefly reprise key principles that contribute to the effectiveness of iodinated CT contrast media. An incident $\mathrm{x}$-ray can undergo photoelectric absorption after interaction with an inner shell electron of an atom in the attenuating medium when the $x$-ray energy is fractionally in excess of the binding energy of the electron (15). 
The subsequent ejection and replacement of the electron from an outer shell results in production of a characteristic $\mathrm{x}$-ray (15).

The photoelectric interaction does not occur when the incident $\mathrm{x}$-ray energy is less than the binding energy of the electron (15). If, however, the incident energy is equal to the binding energy, the photelectric effect becomes possible and a disproportionate increase in attenuation occurs (15). Beyond the binding energy equivalent for the incident $\mathrm{x}$ ray, the probability of photoelectric interaction decreases (15). The K edge refers to the abrupt increase in attenuation when the energy of the incident $\mathrm{x}$-ray approximates the $\mathrm{K}$ binding energy (Fig. 2) (15).

The human body is largely composed of low-atomicmass elements with corresponding low-K shell binding energies (15). Higher atomic masses associated with, among many elements, iodine, gadolinium, and lead have high-K shell binding energies producing characteristic $\mathrm{x}$-rays with relatively high energies (15). This principle allows higheratomic-mass elements to be used for contrast media or detector material (15).

Since the photoelectric effect has a higher probability of occurring between low-energy $\mathrm{x}$-rays and high-atomicmass elements, it provides excellent properties as a CT contrast agent. Iodine, for example, has approximately 350 times higher attenuation than soft tissue at the same energy (15). This property can be exploited in CT contrast imaging. The $\mathrm{K}$ shell binding energy for $\mathrm{x}$-rays is $33.2 \mathrm{keV}$ for

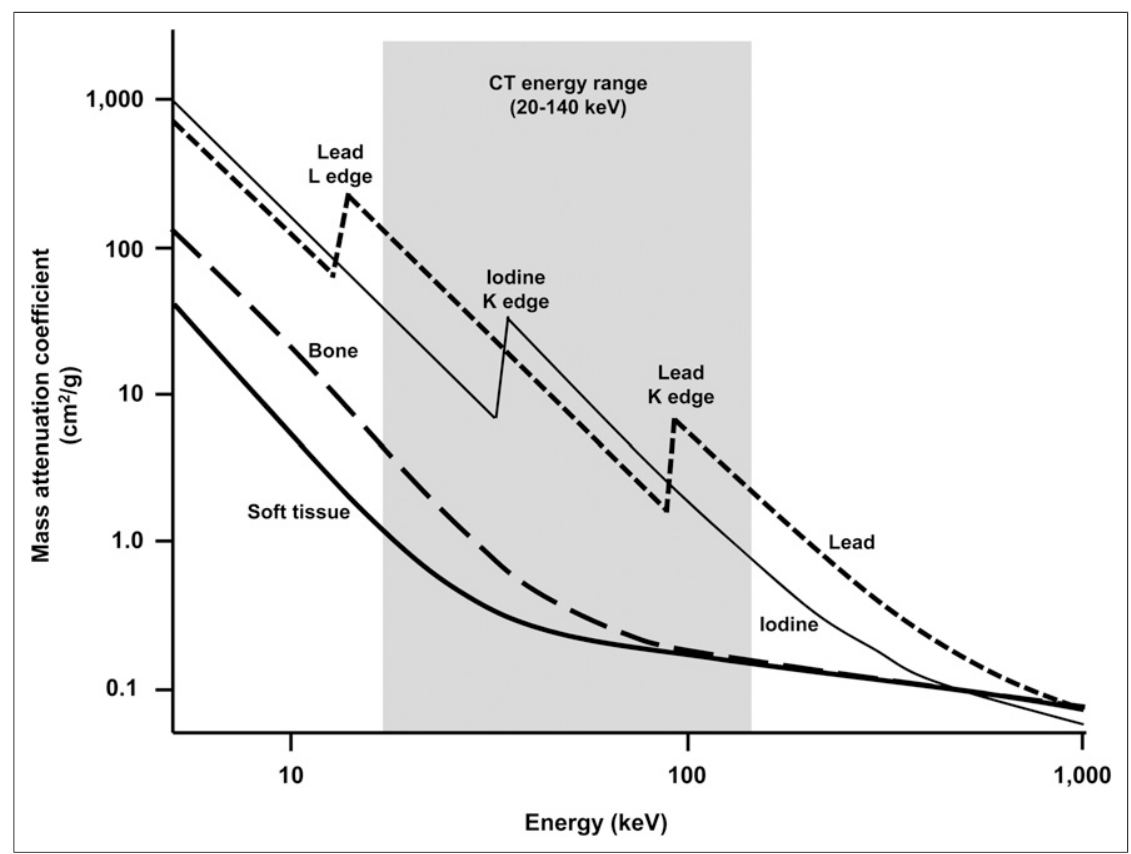

FIGURE 2. Schematic representation on log-log scales of mass attenuation coefficient against x-ray energy. lodine $\mathrm{K}$ edge at $33 \mathrm{keV}$ demonstrates abrupt increase in attenuation that produces equivalent attenuation greater than lead and several orders of magnitude greater than bone and soft tissue. Within range of 30 $100 \mathrm{keV}$, attenuation coefficients for biologic tissues remain fairly uniform whereas contrast agent (iodine) varies substantially. (Adapted from (15).) iodine, but rather than attempting imaging with a monochromatic beam at that energy, $\mathrm{kVp}(60-80)$ can be optimized to produce a good proportion of x-rays in the 33- to $40-\mathrm{keV}$ range. In this energy range, attenuation is greater for iodine than for lead (Fig. 2) (15).

\section{Pharmacokinetics}

Iodinated CT contrast agents are best described using a 2-compartment model (Fig. 3). Iodinated contrast agents demonstrate a rapid peak plasma concentration at $2 \mathrm{~min}$ after intravenous injection (6-8). A biphasic plasma profile represents rapid diffusion of the contrast from the plasma compartment into the interstitial compartment and then slow urinary clearance $(6,8)$. The peak plasma concentration at $2 \mathrm{~min}$ after intravenous injection shortens to $1 \mathrm{~min}$ with higher doses (7). Only 1\%-3\% of iodinated contrast agents undergo plasma protein-binding (8). As contrast diffuses into the extravascular space, water is drawn from the extravascular space into the intravascular space because of osmolality. Tight junctions prevent movement of contrast into extravascular spaces in the brain, testes, and neural tissues.

The biphasic half-lives vary from one agent to the next but tend to be approximately 7 min (range, 2-30 min) and $1.6 \mathrm{~h}$ (range, $1-2 \mathrm{~h}$ ), with these half-lives universally increasing with increasing contrast dose (6-8). In renal dysfunction, elimination half-life can increase to $40 \mathrm{~h}$ or longer (8). The intravenous CT contrast agents do not undergo metabolism and are excreted almost exclusively by glomerular filtration in urine unchanged in a similar fashion to inulin $(6,8)$. The proportion of the contrast dose in urine varies between agents but in healthy individuals is $12 \%$ at $10 \mathrm{~min}, 50 \%$ at $1 \mathrm{~h}, 83 \%$ at $3 \mathrm{~h}$, and virtually $100 \%$ at $24 \mathrm{~h}(6-8)$. Contrast agents may have individual and collective maximum volumes requiring awareness of previous procedures and specifications for the agent in use.

Calcium or magnesium has been added to newer contrast agents to reduce toxicity (12). All ionic contrast media bind in vivo to calcium and magnesium (12). If given orally, CT contrast agents are absorbed from the intestine, glucuroconjugated, strongly plasma proteinbound, and then rapidly concentrated and eliminated in the biliary system (6).

\section{Contraindications and Precautions}

Several medications are relatively contraindicated with ionic contrast media because they will crystalize and form precipitates, including cimetidine, diazepam, diphenhydramine, ethanol, 


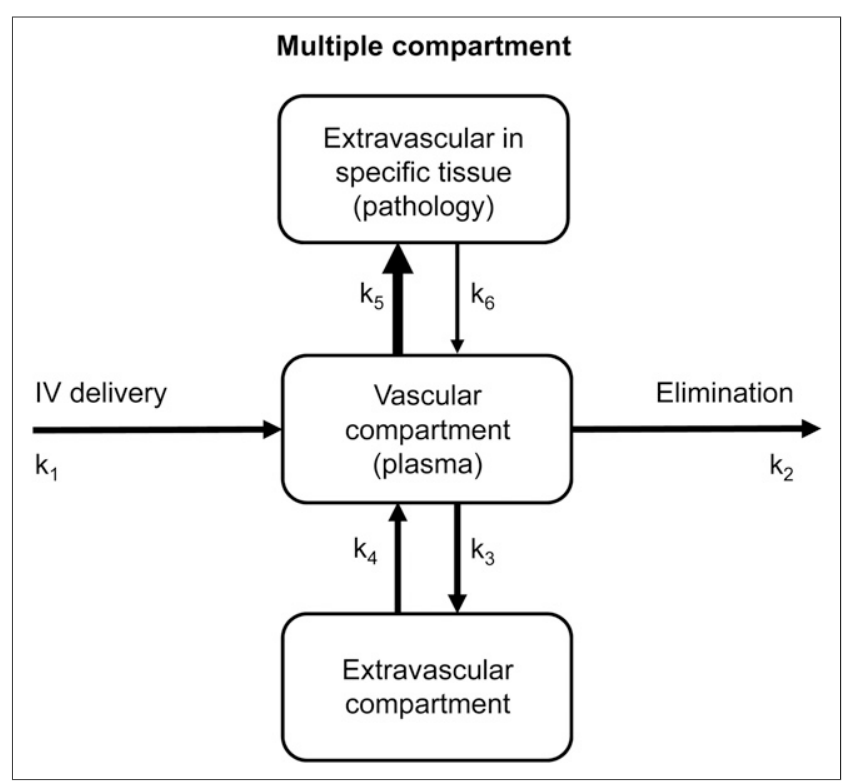

FIGURE 3. Modified 2-compartment model for iodinated CT or gadolinium MRI contrast media administered intravenously. Second extravascular compartment represents pathologic tissue that may enhance with contrast administration and, therefore, be differentiated by surrounding normal tissue by greater rate constant ( $k_{5}$ over $k_{3}$ or $k_{4}$ over $k_{6}$ ). A previous article (2) in this series provides a more detailed interpretation of compartment models and rate constants.

meperidine, papaverine, promethazine, and protamine sulfate $(12,16)$. Clearly, some of these medications are prescribed for prophylaxis or management of adverse reactions and so caution is suggested, especially regarding administration through the same intravenous line. Nonionic contrast agents do not share this incompatibility. Caution should also be exercised when using iodinated contrast agents in patients medicated with known nephrotoxic drugs, including angiotensin-converting enzyme inhibitors, acyclovir, aminoglycosides, amphotericin, antineoplastics, cyclosporin, furosemide, lithium, metformin, methotrexate, NSAIDs, tacrolimus, and vancomycin $(12,16)$. Creatinine levels may warrant assessment before progression with CT contrast agents in these patients. There are numerous factors that identify a patient as having a higher risk of adverse reactions, and although caution should be exercised, none are absolute contraindications. Perhaps the patients at highest risk are those with a GFR of less than $30 \mathrm{~mL} / \mathrm{min} / 1.73 \mathrm{~m}^{2}$. In this group, an iodinated CT contrast agent should not be administered unless the patient is on dialysis and anuric or the diagnostic benefits outweigh the risk. LOCM, nonionic, dimer-based contrast agents with a low iodine concentration are lowerrisk options for patients at increased risk of adverse reactions when the benefit of the contrast procedure warrants iodinated contrast CT.

\section{Adverse Reactions}

Adverse effects are common and classified in several overlapping ways: by type of reaction, severity of reaction, or mechanism of reaction (Fig. 4). Based on a classification of the type of adverse reaction, contrast agents in CT can produce general reactions or organ-specific reactions $(11,16)$. Organ-specific reactions include toxicity associated with renal, cardiovascular, pulmonary, or neurologic systems (11). General reactions can be acute or delayed, with the latter tending to be skin reactions (11). Acute general reactions can be mild and self-limiting or moderate to severe, requiring intervention $(10-12,16)$. If appropriate, adverse reactions should be reported to the regulatory authority. The incidence of adverse reactions to intravenous iodinated contrast agents is higher for ionic than nonionic agents (Table 2) $(10-12,16)$. The most common adverse reactions to $\mathrm{CT}$ iodinated contrast agents are hives $(52.5 \%)$ and nausea $(17.6 \%)$ (13). Prior adverse reactions to CT contrast agents are a significant predictor of a subsequent reaction. There is a prevalence of $17 \%-35 \%$ for adverse reactions to ionic contrast agents in those with previous reactions and $5 \%$ for nonionic contrast agents (11). Other predictors or risk factors for developing an adverse reaction to iodinated intravenous contrast agents include a history of asthma, a history of allergies, heart disease, dehydration, sickle cell disease, polycythemia, myeloma, and underlying renal disease (1113,16). The risks of adverse reactions is also higher in infants and the elderly, during periods of anxiety, and during treatment with medications such as $\beta$-blockers, nonsteroidal antiinflammatories (NSAIDs), and interleukin-2 $(11,13,16)$.

Fatal adverse reactions can occur but are rare (1 in 170,000 cases) (10-12), but with 25 million CT contrast studies performed annually in the United States, that equates to 150 deaths in the United States annually. The risk of a fatal adverse effect increases for women, the elderly, Anglo-Saxons, and those with comorbidity (11). This has been referred to as the 4 W's: women, wrinkled, white, and weakened (11). Generally, the cause of death in fatal CT contrast reactions is renal failure (58\%), anaphylaxis or allergy (19\%), cardiopulmonary arrest $(10 \%)$, respiratory failure (8\%), or stroke or cerebral hypoxia (4\%) (11).

Delayed general adverse reactions occur $1 \mathrm{~h}$ to $1 \mathrm{wk}$ after the intravenous contrast injection (10-12). Delayed reactions tend to be skin reactions and are more likely for dimeric isomolar agents or in young adults, women, and those with a history of allergy $(10,11)$.

The mechanism of adverse contrast reactions can be classified as anaphylactoid, chemotoxic (organ-specific), or vasovagal $(12,17)$. Anaphylactic reactions are associated with an allergen- or IgE-mediated release of chemical mediators such as histamine from mast cells $(12,17)$. Anaphylactoid reactions such as those of iodinated contrast agents are similar to anaphylactic reactions in terms of activation of mast cells but are not initiated by the allergen/IgE complex $(12,13,17)$. This is important to understand because it means that an anaphylactoid CT contrast reaction can occur with the first administration (no sensitization), that reaction severity is not dose-related (test doses are unhelpful), and that a previous history of contrast reaction increases the risk 


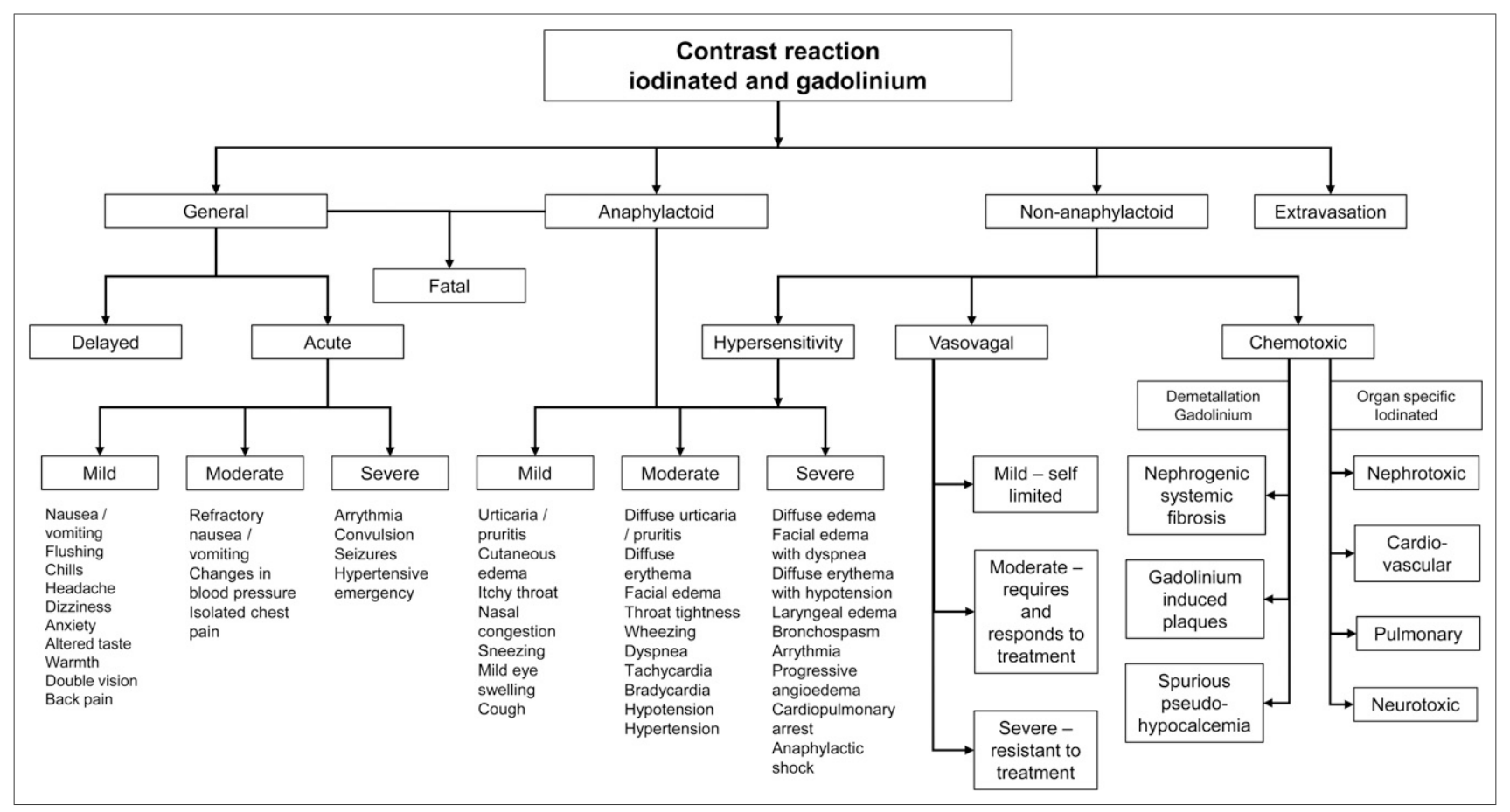

FIGURE 4. Flowchart of iodinated and gadolinium contrast reaction classification.

of a subsequent reaction but does not mean it will occur (17). Nonanaphylactoid reactions are dose-dependent (17). Nonetheless, patients with asthma, food or medication allergies, mastocytosis, or prior contrast reactions are at highest risk of an anaphylactoid reaction $(12,13)$.

Chemotoxic adverse reactions are associated with the ionicity, iodine concentration, viscosity, osmolality, injection rate, and dose of the contrast agent, all contributing to alterations of homeostasis $(12,17)$. Contrast-induced nephrotoxicity lacks a uniform definition around the degree of resulting renal dysfunction, but incidence does vary with baseline renal function status (10-12). There is a 1\%-3\% risk of developing nephrotoxicity in patients with normal baseline renal function, $12 \%-27 \%$ in those with preexisting renal dysfunction, and 50\% in those with diabetic nephropathy $(11,12)$. Preventative strategies include $6-12 \mathrm{~h}$ of prehydration followed by $4-12 \mathrm{~h}$ of posthydration, and the use of a nonionic contrast agent using the minimum dose (11). A $600-\mathrm{mg}$ dose of $N$-acetylcysteine twice daily for $48 \mathrm{~h}$ before the study has been used prophylactically $(10,11)$. A sodium bicarbonate infusion starting $1 \mathrm{~h}$ before contrast administration and continued until $6 \mathrm{~h}$ after contrast administration has also been used (11).

Patient screening based on health history and renal function is perhaps the best strategy for minimizing acute contrast-induced nephrotoxicity. Indeed, estimated glomerular filtration rate (GFR)

TABLE 2

Incidence of Adverse Reactions to lodinated Intravenous Contrast Agents $(10-13,16-18)$

\begin{tabular}{|c|c|c|c|c|}
\hline Reaction type & Ionic & Nonionic & Ionic HOCM & $\begin{array}{c}\text { Nonionic } \\
\text { LOCM/IOCM }\end{array}$ \\
\hline Mild & $15 \%$ & $3 \%$ & & \\
\hline Moderate & $1 \%-2 \%$ & $0.2 \%-0.4 \%$ & & \\
\hline Severe & $0.2 \%$ & $0.04 \%$ & $0.22 \%$ & $0.04 \%$ \\
\hline Fatal & $0.0006 \%$ & $0.0006 \%$ & & \\
\hline Overall & & & $13 \%$ & $3 \%$ \\
\hline Delayed & $\begin{array}{l}2 \%-4 \% \text { for } \\
\text { nonionic } \\
\text { dimer }\end{array}$ & $\begin{array}{l}0.5 \%-1 \% \text { for } \\
\text { ionic and nonionic } \\
\text { monomers }\end{array}$ & $\begin{array}{l}12.5 \% \text { for CT } \\
\text { with intravenous } \\
\text { contrast }\end{array}$ & $\begin{array}{l}10 \% \text { for CT without } \\
\text { intravenous contrast }\end{array}$ \\
\hline Extravasation & $\begin{array}{l}0.04 \%-0.2 \% \text { for } \\
\text { mechanical power } \\
\text { injectors }\end{array}$ & $\begin{array}{l}0.04 \%-0.2 \% \text { for } \\
\text { mechanical power } \\
\text { injectors }\end{array}$ & & \\
\hline $\begin{array}{l}\text { Contrast-induced } \\
\text { nephropathy }\end{array}$ & $\begin{array}{l}1 \%-3 \% \text { in normal } \\
\text { renal function }\end{array}$ & $\begin{array}{l}12 \%-27 \% \text { in renal } \\
\text { impairment }\end{array}$ & $\begin{array}{c}50 \% \text { in diabetic } \\
\text { nephropathy }\end{array}$ & \\
\hline
\end{tabular}


from serum creatinine levels has been used effectively to predict risk. A GFR greater than $60 \mathrm{~mL} / \mathrm{min} / 1.73 \mathrm{~m}^{2}$ is associated with negligible risk of contrast-induced nephrotoxicity (11). Conversely, a GFR of $30-60 \mathrm{~mL} / \mathrm{min} / 1.73 \mathrm{~m}^{2}$ is associated with a moderate risk of contrast-induced nephrotoxicity, and a GFR of less than $30 \mathrm{~mL} / \mathrm{min} / 1.73 \mathrm{~m}^{2}$ is associated with a high risk (relatively contraindicated) (11). Serum creatinine levels should be assessed before CT contrast administration in patients who have a history of renal disease, a family history of renal disease, diabetes, myeloma, or collagen vascular disease or are on medications such as metformin, NSAIDs, and aminoglycosides $(11,12)$. Creatinine assessment is also recommended in patients with a history of renal transplantation, renal tumor, or renal surgery; in patients with end-stage liver disease; and in patients with severe congestive heart failure (12).

The molecular weight of iodinated contrast agents allows ready filtration in the glomeruli. The virtual $100 \%$ elimination of iodinated contrast agents via the glomeruli creates potential for nephrotoxicity. Iodinated contrast nephrotoxicity is generally associated with several mechanisms (Fig. 5). First, hypertonic solutions in the renal tubules reduce water reabsorption, thus leading to tubular swelling, increased intrarenal pressure, and a decrease in both renal blood flow and GFR (12). Concurrently, increased viscosity increases tubular pressure. Second, tubular cell damage causes a decrease in clearance of paraaminohippurate and an increase in excretion of enzymes in the proximal tubules. Medullary hypoxia resulting from vasoconstriction and decreased blood flow also contribute to tubular cell damage. Tubular cell damage both reduces GFR and increases oxidative stress. Third, cytotoxicity damages the endothelium, causing vasoconstriction and decreased GFR, and increases oxidative stress. Vasoconstriction can be exacerbated in patients who have diabetes or are using vasoconstrictive drugs (12).

Cardiovascular toxicity results in an increased incidence or severity of cardiovascular adverse reactions to iodinated contrast agents. Underlying heart disease increases the risk of cardiovascular toxicity (16). Neurotoxicity associated with iodinated contrast agents results from an alteration in the blood-brain barrier due to the hypertonicity (16). Clearly, LOCM and IOCM agents reduce this risk. Headache, confusion, seizures, altered consciousness, visual disturbances, and dizziness are the most common signs of neurotoxicity.

Vasovagal reactions manifest as bradycardia and hypotension because the involuntary reflex slows heart rate and dilates blood vessels in the legs (12). Vasovagal reactions are not necessarily attributed to the contrast agent itself but are a sympathetic nervous system response to fear or pain (12). Clearly, anxiety can exacerbate this. With more blood in the legs and less in the brain, patients may get lightheaded and faint. Elevating the patients' legs in combination with administering 6-10 L of oxygen per minute are generally adequate treatment.

Life-threatening reactions usually occur in the first $20 \mathrm{~min}$ after intravenous contrast injection (12). Indeed, 94\%-100\%

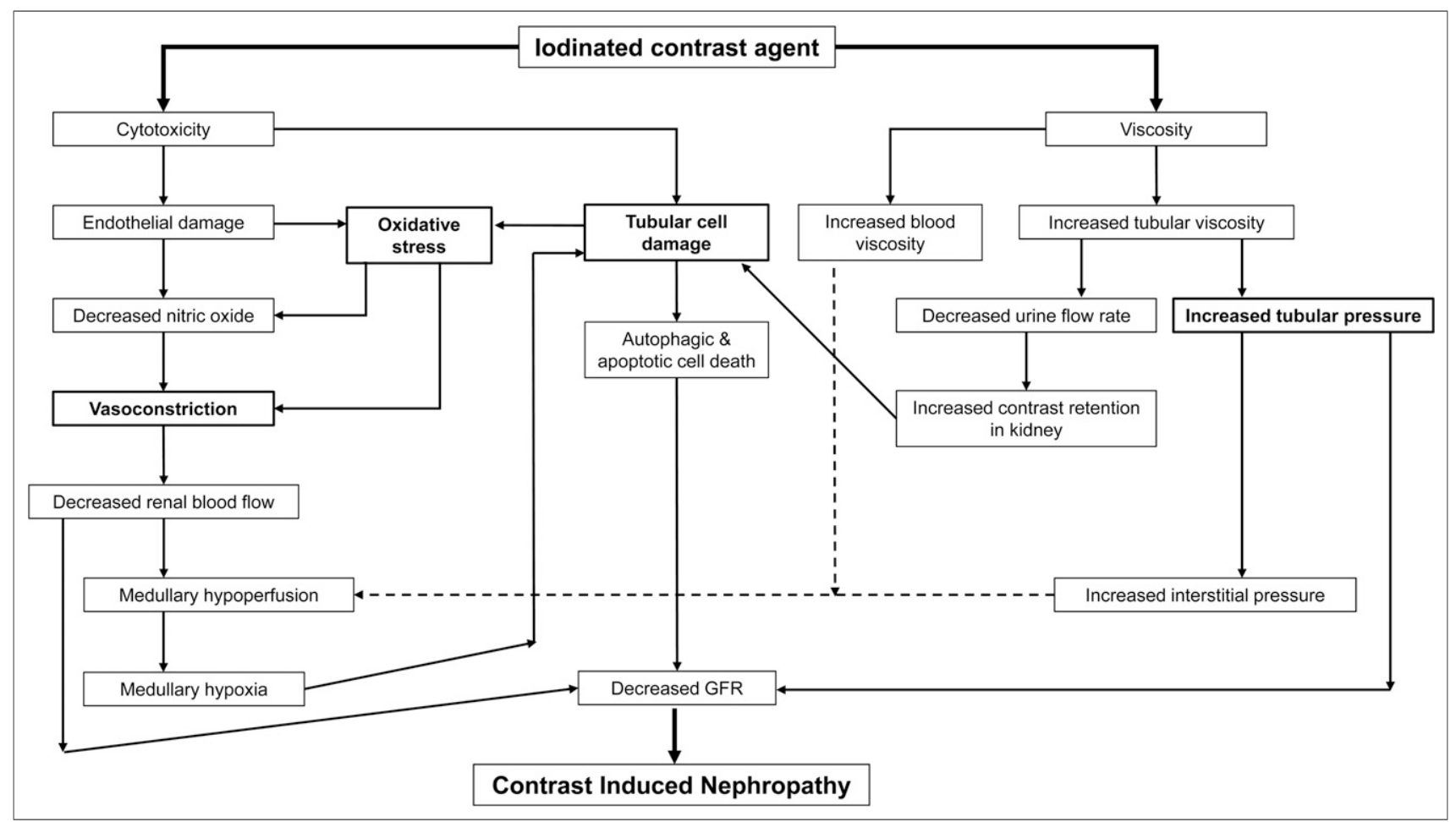

FIGURE 5. Flowchart outlining interplay between factors contributing to development of contrast-induced nephrotoxicity. As outlined by bold boxes, vasoconstriction, oxidative stress, tubular cell damage, and increased tubular pressure are key drivers associated with cytotoxicity and viscosity as mediators. 
of severe or fatal adverse reactions to iodinated CT contrast agents occur within 20 min of administration (13).

\section{Management of Adverse Reactions}

Prevention is better than cure. Prophylactic medications to reduce but not necessarily eliminate risk include $32 \mathrm{mg}$ of methylprednisolone orally $12 \mathrm{~h}$ and $2 \mathrm{~h}$ before contrast administration (Lasser protocol) and $50 \mathrm{mg}$ of diphenhydramine orally $1 \mathrm{~h}$ before contrast administration plus $50 \mathrm{mg}$ of prednisone orally $13 \mathrm{~h}, 7 \mathrm{~h}$, and $1 \mathrm{~h}$ before contrast administration (Greenberger protocol) $(11,13,17,18)$. The Lasser protocol reduces adverse reactions from $9 \%$ to $6.4 \%$ for ionic contrast agents, whereas the Greenberger protocol has been reported to decrease the incidence of adverse reactions to ionic contrast agents from $9 \%$ to $7 \%$ (13). Antihistamines are often used prophylactically in high-risk patients, without evidence of being able to reduce the incidence of adverse reactions (13).

An emergency crash cart should be immediately available at all times in or immediately adjacent to the CT room where intravenous contrast is being used. One should also keep in mind that serious adverse effects may occur in the short period after the patient leaves the scanning room.

Salbutamol (albuterol) has been previously detailed (5) and functions as a $\beta_{2}$-receptor agonist to cause bronchodilation and relieve bronchospasm. The standard dose is 1-2 inhalations of $100 \mu \mathrm{g}$ each, with a third inhalation if necessary $1 \mathrm{~min}$ after the second $(16,17,19)$. This same dose can be given prophylactically. Patients with bronchospasm should also be supported with $10 \mathrm{~L}$ of oxygen per minute delivered via a mask $(13,17)$. Atropine is a parasympatholytic agent that can be used to treat bradycardia in a vasovagal reaction. The standard dose is $0.6-1.0 \mathrm{mg}$ intravenously, repeated every 3-5 min as needed to a maximum of $3 \mathrm{mg}$ $(13,16-19)$. Elevation of the patient's legs and delivery of oxygen $(10 \mathrm{~L} / \mathrm{min})$ should be used to support the self-limiting vasovagal patient $(13,17)$. Diphenhydramine (Benadryl; Johnson and Johnson) is a histamine-1 inhibitor; however, antihistamines (4) block further histamine-mediated reactions but do not stop histamine-mediated reactions already under way. Diphenhydramine should be used only for mild urticaria or prophylaxis, with doses of 25-50 mg orally or intramuscularly, or $25 \mathrm{mg}$ intravenously being standard $(13,17)$. This dose can cause drowsiness. Epinephrine (adrenaline) is a sympathetic agonist with $\alpha$ - and $\beta$-receptor activity. $\alpha$-receptor agonism with epinephrine causes peripheral vasoconstriction, which can help with severe urticaria, facial edema, and laryngeal edema $(13,16,19,20)$. $\beta_{1}$-receptor agonism produces inotropic and chronotropic effects (5) so should be used with caution in known heart disease. Because $\beta_{2}$-receptor agonism causes bronchodilation, epinephrine can be used to treat bronchospasm $(13,16,17,19,20)$. Doses of epinephrine are typically $0.1-0.3 \mathrm{mg}(1 \mathrm{mg} / \mathrm{mL}$ or $1: 1,000)$ subcutaneously/0.3 mg intramuscularly via EpiPen (Mylan Inc.) if the patient is not hypotensive, or $0.1 \mathrm{mg}(0.1 \mathrm{mg} / \mathrm{mL}$ or $1: 10,000)$ intravenously over 3-5 min and repeated as needed up to a maximum of
$1 \mathrm{mg}$ if the patient is hypotensive $(13,16,17,19,20)$. Diazepam is a benzodiazepam that can be used to treat seizures if needed (4), using an intravenous dose of 5-10 $\mathrm{mg}$ to a maximum of $30 \mathrm{mg}$ as required. Lorazepam is an alternative benzodiazepam for seizures, especially in pediatric patients, with a dose of $0.01 \mathrm{mg} / \mathrm{kg}$ intravenously. Nitroglycerin (5) is a vasodilator that decreases oxygen demand and can be used to treat acute angina. The dose can be any one of the following: one 300- to $600-\mu \mathrm{g}$ sublingual tablet under the tongue, 1 or 2 sprays of $400 \mu \mathrm{g}$ each directed onto or under the tongue, or a 2- or 3-mg buccal tablet placed between the upper lip and gum. Sublingual-tablet and spray doses can be repeated if necessary.

In the case of generalized anaphylaxis-like symptoms, epinephrine should be used. Doses are typically $0.1-0.3 \mathrm{mg}$ (1 mg/mL or $1: 1,000)$ subcutaneously/0.3 mg intramuscularly via EpiPen if the patient is not hypotensive, or $0.1 \mathrm{mg}$ $(0.1 \mathrm{mg} / \mathrm{mL}$ or $1: 10,000)$ intravenously over $3-5 \mathrm{~min}$ and repeated as needed up to a maximum of $1 \mathrm{mg}$ if the patient is hypotensive $(13,16,17,19,20)$. A $\beta_{2}$-agonist can be added for bronchospasm. Saline infusion should be used for hypotension.

Nausea and vomiting are self-limiting, and patients need to be observed for 30 min while keeping intravenous access open (13). If nausea and vomiting continue, antiemetic medications can be considered (13). Antiemetics are a class of medication that block neurotransmitters (e.g., acetylcholine, histamine, dopamine, substance $\mathrm{P}$, and 5-hydroxytryptamine) responsible for nausea and vomiting in the emetic center in the medulla, vestibular apparatus, chemoreceptor trigger zone in the fourth ventricle, and higher brain centers that relay sensory inputs (19). Specific examples of medications include metoclopramide for $\mathrm{D}_{2}$ receptor antagonism, hyoscine hydrobromide for muscarinic receptor antagonism, promethazine for histamine-1 receptor antagonism, ondansetron for $5-\mathrm{HT}_{3}$ receptor antagonism, and aprepitant for $\mathrm{NK}_{1}$ receptor antagonism (19). The recumbent position helps minimize aspiration.

\section{Extravasation}

Extravasation of iodinated contrast agents involves delivery of the agent extravascularly because of human error, canula dislodgement, or leakage. Although extravasation is well recognized, its true incidence is hard to reliably determine, but several large studies have reported an incidence of less than $1 \%$ (10). Extravasation of iodinated contrast agents typically causes self-limiting symptoms such as pain, erythema, and swelling (21). In severe reactions, skin ulceration and necrosis, or the development of compartment syndrome, may occur $(21,22)$. Although more severe reactions tend to occur with larger volumes of extravasated HOCM or ionic contrast media, they can occur with small volumes of LOCM and nonionic contrast media (21). The peak reactions occur at $24-48 \mathrm{~h}$ after intravenous contrast administration.

Once again, prevention is a better option than therapy. Some key strategies to minimize the risk of extravasation 
include having reliable intravenous access that is tested before contrast administration, decreasing flow rates if the protocol allows in high-risk patients, limiting flow rates to $3 \mathrm{~mL} / \mathrm{s}$ for large veins and $1.5 \mathrm{~mL} / \mathrm{s}$ for the hand or wrist, monitoring the infusion site directly for the first $15 \mathrm{~s}$ of the infusion, communicating with the patient to report immediately any unusual sensations at the intravenous site during contrast administration, and immediately stopping the infusion if there is concern about extravasation $(23,24)$.

If extravasation of iodinated contrast agents does occur, management will depend on the patient symptoms and volume of extravasation. The infusion should be stopped immediately, and the intravenous site should be elevated and a cold compress applied $(11,25)$. For small volumes and self-limiting symptoms, the patient should be monitored in the CT department for 2-4 h (11,25). For large volumes (30-100 mL), blistering or ulceration, altered perfusion, a change in sensation, or worsening pain or swelling after the 2- to 4-h monitoring window, the patient should be referred to the local emergency department for surgical consultation $(11,25)$.

\section{Interactions}

Iodinated contrast agents are not highly active pharmacologically; however, interactions with medications the patient may be taking are possible (26). Prevention is a good practice and can be achieved by taking a thorough patient history, being aware of drugs needing precautions, and not administering contrast agents through the same line as medications (26). Because iodinated contrast agents have anticoagulant properties (less so for nonionic agents) and prolong coagulation time, they can potentiate the effects of anticoagulant medication (e.g., heparin and warfarin), antiplatelet medication (e.g., aspirin and NSAIDs), and fibrinolytic medication (e.g., urokinase) $(12,16,26)$. Metformin can have additive effects associated with iodinated contrast toxicity $(12,16,26)$. $\beta$-blockers increase the risk and severity of anaphylactoid reactions $(12,16)$. Calcium channel blockers can potentiate the hypotensive effects of iodinated contrast agents $(12,16)$. Diuretics can have a cumulative effect with iodinated contrast agents for diuresis and increase the risk of nephrotoxicity $(12,16,26)$. Nephrotoxic medications such as NSAIDs and gentamicin can potentiate the renal effects of iodinated contrast agents (26). Adverse reactions to iodinated contrast agents are more likely if the patient is taking immunomodulator medications $(12,16,26)$. Allergic reactions or symptoms of a similar nature are more likely in patients taking $\beta$-blockers, interleukins, and interferons (26). Patients taking $\beta$-blocker medications have a 3 -fold increased risk for anaphylactoid adverse reactions to iodinated contrast agents (26). Any medication that relies on renal elimination may have increased retention and activity, and those medications with a narrow therapeutic index may be susceptible to toxicity (26). Synergistic effects between iodinated contrast media and calcium channel blockers and digoxin are possible, especially with ionic HOCM (26).

\section{MRI CONTRAST MEDIA}

\section{Introduction}

There are several different types of contrast media used in MRI; however, most of those used clinically and the focus of this article are the T1 paramagnetic contrast agents associated with gadolinium. Worldwide, 40\%-50\% of MRI procedures are undertaken with contrast agents (27). Nonetheless, it is important to highlight key aspects of the 5 classes of MRI contrast agents (28).

T1 Agents. T1 agents are discussed in more detail below.

T2/T2* Agents. T2/T2* agents are superparamagnetic nanoparticles comprising iron oxides that shorten the relaxation times of T2/T2* (28). Although paramagnetic contrast agents increase the proton signal, superparamagnetic/ ferromagnetic contrast agents destroy the signal (negative contrast) (28). For example, MRI contrast might be achieved by a T2/T2* contrast agent destroying the signal from liver but not from a liver metastasis.

Chemical Exchange Saturation Transfer Agents. Chemical exchange saturation transfer agents are chemicals that create MRI contrast by transferring saturated protons to the bulk water pool (28).

${ }^{19} \mathrm{~F}$ Nuclei. ${ }^{19} \mathrm{~F}$ nuclei are not only naturally occurring fluorine but also the most sensitive spin (resonance) in MRI after hydrogen (28). As a result, ${ }^{19} \mathrm{~F}$ nuclei are readily detected by MRI (28). The barrier to using ${ }^{19} \mathrm{~F}$ nuclei instead of ${ }^{18} \mathrm{~F}$ nuclei in radiopharmaceuticals is that in nuclear medicine the tracer principle is adopted whereas MRI requires large (potentially toxic) concentrations: 1 fluorine atom versus 20 or more per structure. Instead, ${ }^{19} \mathrm{~F}$ nuclei are incorporated into perfluorocarbon nanoparticles, avoiding toxicity by encapsulation of many perfluorocarbon molecules in a phospholipid-encapsulated nanoparticle.

Hyperpolarized Probes. Hyperpolarized probes use polarization techniques (such as dynamic nuclear polarization) to increase (up to 5 orders of magnitude) sensitivity to the spin energy levels (28). Unfortunately, despite excellent sensitivity, these agents require fast injection and target accumulation to overcome signal decay (28).

Paramagnetic substances have one or more particles (protons, neutrons, or electrons) with a spin that is not cancelled by another similar particle with an opposite spin. Because magnetic dipole moments of unpaired electrons are much larger than those of protons or neutrons, the local magnetic fields generated by unpaired electrons are strong (28). Substances that have unpaired electrons, such as gadolinium, are effective paramagnetic contrast enhancers (28). When paramagnetic ions are added to water, the relaxation of water molecules is enhanced near the paramagnetic substance $(27,28)$. Both T1 and T2 relaxation times are then reduced. It is not the actual contrast agent that alters the intensity of the image but the presence of the contrast agent that alters the relaxation characteristics of adjacent protons, thus indirectly affecting the intensity (28). Although gadolinium is technically a paramagnetic agent as a result of its unpaired 
electrons, this characteristic is not the basis of $\mathrm{T} 1$ contrast enhancement $(28,29)$. It is the dipole-dipole interaction that influences T1 relaxation (27-29). Gadolinium contains 7 unpaired electrons, making these compounds strongly paramagnetic (27). Gadolinium is a lanthanide because the inner electron shells of its atomic structure are not filled. In its ionized form $\left(\mathrm{Gd}^{3+}\right)$, gadolinium donates electrons from other subshells, leaving the seven $4 \mathrm{f}$ electrons unpaired, and this property ensures paramagnetic behavior after chelation.

\section{Properties}

Gadolinium contrast agents have several key properties that influence their behavior, efficiency, and adverse reaction profile. Interplay among these properties optimizes the degree of enhancement and its tolerability. These properties include chemical structure, osmolality, viscosity, iconicity (not to be confused with demetallation [dechelation]), relaxivity, half clearance rate, and dose (Table 3) (29).

Gadolinium is tightly chelated into a complex chemical structure that can be linear or macrocyclic (Fig. 6). Macrocyclic chemical structures use DOTA chelation. The chemical structure prevents heavy-metal toxicity because the tight chelation prevents cellular uptake of the toxic free gadolinium ion (27). Collectively, the chemical structure and chelation of gadolinium enhance renal elimination and maintain distribution in the extravascular space $(27,29)$. Regardless of chemical structure, the pharmacodynamics and pharmacokinetics are the same (29). The standard gadolinium contrast dose is $0.1 \mathrm{mmol} /$ $\mathrm{kg}(27,29)$. This dose primarily influences the $\mathrm{T} 1$ relaxation time, but higher doses may increase the effect of $\mathrm{T} 2$ relaxation.

Relaxivity measures the degree to which a given amount of contrast agent shortens $\mathrm{T} 1$ or $\mathrm{T} 2$, and higher relaxivity equates to better enhancement. Relaxivity refers to the contrast agents' change in relaxation rates with changes in concentration. Largely, the early-generation gadolinium contrast agents shared the same T1 relaxivity whereas later-generation contrast agents have up to $50 \%$ higher $\mathrm{T} 1$ relaxivity at $1.5 \mathrm{~T}(29)$.

As with iodinated contrast agents, osmolality contributes to the incidence of nonanaphylactoid adverse reactions mediated by endothelial damage, movement of fluid among compartments, and cell deformation. Adverse effects attributable to osmolality include pain, flushing, nausea, and vomiting (12). Contrast osmolality higher than blood results in movement of water from interstitial spaces into the vascular compartment, which causes increased blood viscosity, endothelial damage, hypervolemia, vasodilation, edema with neurotoxicity, decreased myocardial contractility, and toxicity $(6,29)$. Gadolinium contrast agents are administered in much lower doses than iodinated agents in $\mathrm{CT}$, and as such, the alteration to plasma osmolality is very low, reducing the impact of adverse reactions by comparison (27). Nonetheless, when large gadolinium contrast doses are to be administered, low-osmolality agents should be used (27).

The viscosity of gadolinium contrast agents is the flow friction, resistance, or thickness of the contrast medium $(8,12)$. Gadolinium contrast agents are generally of similar viscosity to blood at body temperature $\left(37^{\circ} \mathrm{C}\right)(29)$. Viscosity plays an important role in renal tolerance, with near-serum viscosity reducing the risk of contrast-induced nephrotoxicity associated with iodinated contrast media. The viscosity of gadolinium contrast agents is not considered a significant concern for adverse reactions $(27,29)$.

Ionic gadolinium CT contrast agents dissociate into ion pairs whereas nonionic agents do not (29). Ionic agents may increase the risk of some adverse reactions such as cardiac arrhythmia (29). A combination of ionic and high osmolar gadolinium agents increases the adverse cardiovascular effects, and the hemodynamic changes present a risk to patients with coronary artery disease (29). The low dose of gadolinium contrast agents compared with iodinated CT contrast agentsor even compared with ionic MRI contrast agents-causes the ionic charge of the gadolinium complex to not be considered significant in terms of safety and risk of adverse reactions (27).

\section{Mechanism of Action}

As with iodinated CT contrast agents, MRI contrast agents provide image contrast without altering biologic function (29). Unlike iodinated contrast agents in CT, MRI contrast

TABLE 3

Comparison of Key Properties of Main Gadolinium Contrast Agents

\begin{tabular}{|c|c|c|c|c|c|c|}
\hline Contrast agent & Structure & Ionicity & Clearance $\mathrm{T}_{0.5}(\mathrm{~h})$ & $\begin{array}{c}\text { Osmolality } \\
\text { (mOsm/kg water) }\end{array}$ & $\begin{array}{l}\text { Viscosity } \\
\text { at } 37^{\circ} \mathrm{C}(\mathrm{cP})\end{array}$ & $\begin{array}{l}\text { T1 relaxivity } \\
(\mathrm{L} / \mathrm{mmol}-\mathrm{s})\end{array}$ \\
\hline Gadopentetate dimeglumine & Linear & Ionic & 1.6 & 1,960 & 2.9 & 4.1 \\
\hline Gadoteridol & Macrocyclic & Nonionic & 1.57 & 630 & 1.3 & 4.1 \\
\hline Gadodiamide & Linear & Nonionic & 1.3 & 789 & 1.4 & 4.3 \\
\hline Gadoversetamide & Linear & Nonionic & 1.73 & 1,110 & 2.0 & 5.2 \\
\hline Gadobenate dimeglumine & Linear & Ionic & $1.2-2$ & 1,970 & 5.3 & 6.3 \\
\hline Gadoterate & Macrocyclic & Ionic & 1.6 & 1,350 & 2.4 & 3.6 \\
\hline Gadobutrol & Macrocyclic & Nonionic & 1.81 & 1,603 & 5.0 & 5.2 \\
\hline Gadoxetate & Linear & Ionic & 0.93 & 688 & 1.2 & 6.9 \\
\hline Gadofosveset & Linear & Ionic & & 1,110 & 3.0 & 19 \\
\hline Blood & & & & 290 & $1.5-2$ & \\
\hline
\end{tabular}

With exception of gadoxetate, dose is $0.1 \mathrm{mmol} / \mathrm{kg}$. Gadoxetate dose is $0.025 \mathrm{mmol} / \mathrm{kg}(27,29,31)$. 


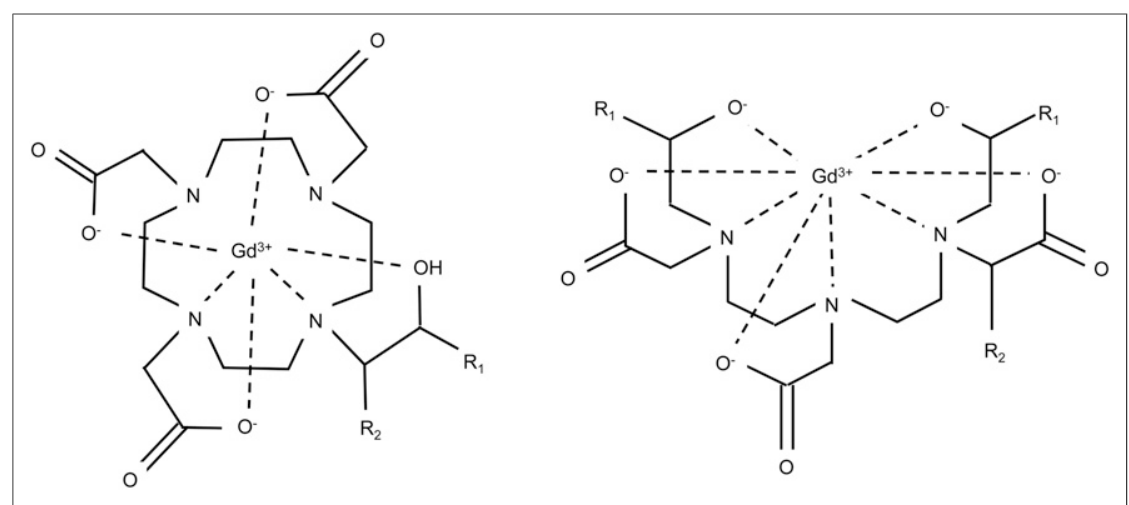

FIGURE 6. Chemical structure of gadolinium contrast agents adopts either macrocyclic base (left) or linear base (right), with major differences between each agent being changes to $\mathrm{R}$ groups.

agents are detected not directly but rather indirectly by influencing the nuclear magnetic relaxation time of water $(27,29)$. Although gadolinium shortens the relaxation time constants for $\mathrm{T} 1, \mathrm{~T} 2$, and $\mathrm{T} 2 *$ in adjacent protons (water) in tissues, it is the shortening of $\mathrm{T} 1$ that is the target of MRI $(27,29)$. As a result, rapid acquisition of T1-weighted MR images will have an increased signal from contrast-enhanced tissues (29). MRI physics is too complex to describe in the context of this article, but some assumed knowledge is necessary to understand the mechanism of action. Nonetheless, Figure 7 provides a schematic representation of $\mathrm{T} 1$ and $\mathrm{T} 2$ contrast enhancement. Specifically, gadolinium causes T1 shortening through dipole-dipole interactions with protons $(27,29)$.

\section{Pharmacokinetics}

Gadolinium is a heavy-metal toxin when free in vivo as an ion, yet the risk of adverse reactions is low and most are mild (29). This is primarily the result of the chemical stability associated with gadolinium chelation (much the same way ${ }^{68} \mathrm{Ga},{ }^{99 \mathrm{~m}} \mathrm{Tc}$, and other radiometals are chelated in radiopharmaceuticals). These chelates also overcome the potential consequences of the long biologic half-life of free gadolinium (29). Consequently, gadolinium contrast agents are almost exclusively eliminated unchanged (no metabolism) via the kidneys, with a half-clearance time in normal renal function of 1.5-2 h (29). Renal clearance occurs without secretion or reabsorption $(27,30)$. Gadolinium contrast demonstrates $85 \%$ elimination by $4 \mathrm{~h}$ after intravenous administration, $98 \%$ elimination by $24 \mathrm{~h}$, and $100 \%$ by $48 \mathrm{~h}$, but renal dysfunction prolongs retention $(27,30)$.

Extracellular gadolinium chelates have pharmacokinetic properties similar to iodinated CT contrast media $(27,30)$. After intravenous administration, gadolinium contrast agents are rapidly cleared from intravascular space to extravascular space, with a distribution equivalent to extracellular water $(27,30)$. They show no plasma protein binding and do not cross intact blood-brain barrier $(27,30)$. Like iodinated contrast agents, gadolinium clearance is biphasic, with the initial distribution having a 4-min half-life followed by the aforementioned 1.5 - to 2 -h elimination half-life
$(27,29,30)$. Gadolinium contrast agents are best illustrated with a 2-compartment model as illustrated for iodinated contrast agents (Fig. 3). Gadolinium contrast agents can enter and wash out from normal tissues and diseased tissues at different rates, which will change the $\mathrm{T} 1$ and $\mathrm{T} 2$ relaxation times between those tissues $(27,30)$.

\section{Contraindications and Precautions}

Gadolinium contrast agents cross the placenta and undergo fetal excretion via the kidneys. The excreted agent remains in the amniotic fluid for a protracted period during which it can undergo demetallation (dechelation) into free gadolinium and expose the fetal lungs and gut (29). Consequently, gadolinium contrast agents are contraindicated during pregnancy, especially the first trimester (27). If gadolinium contrast agents are to be applied during pregnancy, macrocyclic varieties should be used (27). Although gadolinium contrast agents are also excreted in breast milk, the dose to the infant is a fraction of that to the mother and is excreted after oral absorption in feces (29). There is no need for cessation of breast feeding (27), although a cautious approach would include expressing and discarding for $6 \mathrm{~h}$ after intravenous gadolinium contrast injection.

Although gadolinium contrast agents are considered safe and biologically inert, they are more nephrotoxic than iodinated contrast agents in the equivalent dosage (27). High doses in patients with impaired renal function are contraindicated $(16,27)$. Acute renal failure occurs in $3.5 \%$ of cases of abnormal creatinine levels (27). Gadolinium contrast agents are contraindicated in patients with a GFR below $30 \mathrm{~mL} / \mathrm{min} /$ $1.73 \mathrm{~m}^{2}$ or with acutely deteriorating renal function (16). Patients with previous anaphylactoid or hypersensitivity reactions to gadolinium contrast agents should be treated with caution (16). Preexisting nephrogenic systemic fibrosis (NSF) is also a contraindication (16). Caution should be exercised in gadolinium contrast use in patients with moderate renal dysfunction, epilepsy, hypotension, a history of hypersensitivity, asthma, or allergic respiratory disorders (16). Caution should be exercised in patients with severe cardiovascular disease or drug-induced arrythmia (16).

\section{Adverse Reactions}

Adverse effects to gadolinium contrast agents are uncommon, with an incidence generally reported in the range of $1.5 \%-2.4 \%(27,29)$. Most adverse reactions are considered mild and more commonly include mucosal reactions, urticaria, vomiting, change in taste, local warmth, local pain, headache, paresthesia, and dizziness (Fig. 4) (16,27,29,31). Vasodilation and injection site discomfort are also possible (29). The risk of an adverse reaction increases in patients 


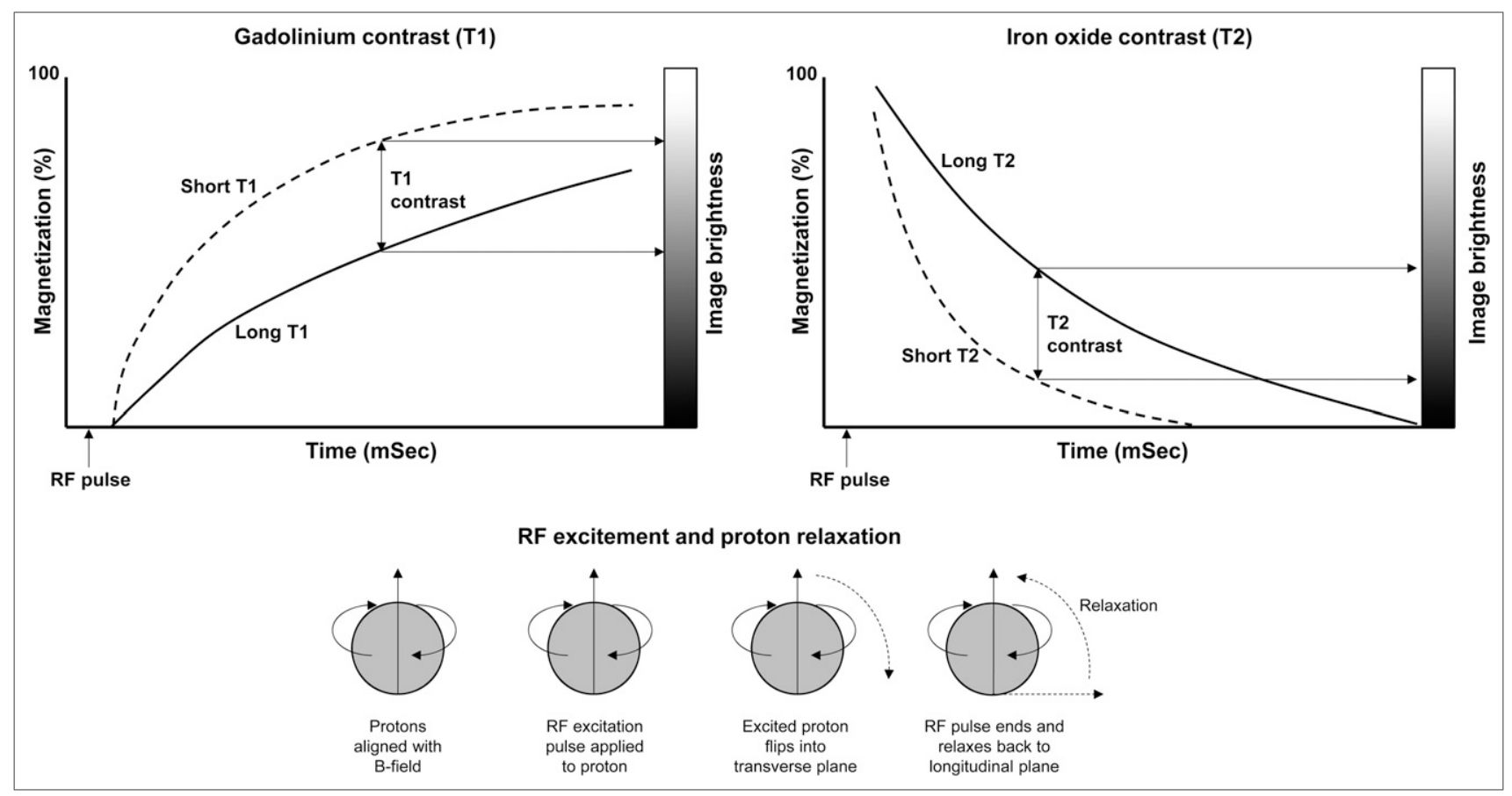

FIGURE 7. Schematic representation of principle of T1 and T2 contrast enhancement in MRI. As represented at bottom of figure, hydrogen (protons) initially aligns with magnetic field. Radiofrequency (RF) excitation pulse is applied to proton, which flips into transverse plane. RF pulse ends, allowing proton to relax back to longitudinal plane. T1 plot (top left) shows effect of shortening relaxation time with gadolinium contrast and resultant positive enhancement of contrast. Likewise, T2 plot (top right) shows effect of shortening relaxation time with iron oxide contrast and resultant negative enhancement of contrast.

with asthma, allergies, and a history of contrast reaction to gadolinium or iodine $(27,29)$. If appropriate, adverse reactions should be reported to the regulatory authority.

In a study of 17,767 patients undergoing cardiac MRI with gadolinium contrast, only $0.17 \%$ of patients experienced adverse reactions, and $100 \%$ of those were mild (32). Furthermore, the adverse reaction rate between different agents varied between $0.06 \%$ and $0.47 \%$ (32). In another study, with 194,400 ionic, linear gadolinium contrast injections, there was a $0.1 \%$ incidence of adverse reactions, of which $83.8 \%$ were classified as mild, $13.7 \%$ moderate, and $2.4 \%$ severe (5 cases) (33).

Acute hypersensitivity reactions occur within $1 \mathrm{~h}$ of contrast administration and in $0.1 \%$ of patients and include mild pruritis (itching) and urticaria (hives) (34). A prior hypersensitivity reaction to a gadolinium contrast agent is associated with a $30 \%$ risk of reaction to subsequent gadolinium administrations and with greater severity (34). Patients can experience moderate hypersensitivity reactions, which include bronchospasm, laryngeal edema, facial edema, tachycardia, angioedema, hypotension, arrhythmia, and widespread urticaria $(16,34)$. Severe anaphylactoid reactions have an incidence of $0.005 \%$, and fatal reactions, $0.0003 \%$. An increased risk of a hypersensitivity reaction to gadolinium contrast agents exists for female patients, patients with allergies or asthma, and patients who had prior gadolinium contrast administrations (34).

The adverse reaction profile of gadolinium contrast agents is uniform across the range of agents $(27,29)$. This uniformity may seem a little surprising given the variability in ionicity, viscosity, and osmolality, which are known to be major contributors to adverse reactions in iodinated contrast media. This uniform reaction profile is likely to reflect actual differences that cannot be distinguished statistically. For example, there is a 10-fold difference in the incidence of urticaria $(0.2 \%-2 \%)$ across a range of gadolinium contrast media, but the data do not permit a statistically significant difference to be reported (29). Likewise, the incidence of nausea ranges from $1.2 \%$ to $3.2 \%$ across the range of contrast agents (29). Faster injection rates (e.g., MRI angiography) have been reported to increase the incidence of nausea (29). Acute reactions occur even with antihistamine or corticosteroid premedication (33).

Gadolinium MRI contrast agents are not generally considered nephrotoxic, because of the low viscosity and comparatively low dose $(27,29)$. Contrast-induced nephrotoxicity for iodinated contrast agents (Fig. 5) is driven by viscosity and osmolality. Gadolinium contrast agents have near-serum viscosity and sufficiently low doses that blood and tissues remain isotonic after contrast administration. Gadolinium contrast agents do, however, have higher nephrotoxicity than iodinated CT contrast agents for an equivalent dose (27).

Free gadolinium $\left(\mathrm{Gd}^{3+}\right)$ is highly toxic, as relates, in part, to cellular inhibition of calcium $\left(\mathrm{Ca}^{2+}\right)$ at calcium channels $(27,30,35)$. As outlined in a previous article in this series (5), alteration of calcium influx interferes with muscle contraction (e.g., cardiac force of contraction), vascular smooth muscle vasoconstriction, and bronchial smooth 
muscle bronchoconstriction. Free gadolinium can also depress the reticuloendothelial system, including inhibition of phagocytosis $(27,30)$. The median lethal dose for nonchelated gadolinium is $0.35 \mathrm{mmol}$ of whole DOTA per kilogram; chelation increases this to $10.6 \mathrm{mmol} / \mathrm{kg}$ (30). Recent investigations have shown increased signal intensities in the dentate nucleus and globus pallidus suggesting concentration of free gadolinium (35). The clinical and adverse implications of this finding are currently unclear.

Gadolinium has an excellent safety record; however, patients are at risk of developing NSF $(29,36)$. Unfortunately, NSF is irreversible with no treatment available and is associated with progressive movement, swallowing, and breathing difficulties $(29,36,37)$. NSF patients may develop subcutaneous edema causing hard, erythematous plaques of skin with or without hyperpigmentation, papules, blistering, and ulceration (37). Patients present with symptoms of pain, severe pruritus, paresthesia, flexion contractures, and unstable hypertension (37). The incidence of NSF is low and has decreased substantially since the risk became known. An evaluation of over 185 million gadolinium contrast injections revealed less than 1,000 cases of NSF $(0.0005 \%)$ (37). This risk varied among contrast agents from 1 in 2 million to 1 in 50,000 (37). Linear nonionic chelates are considered to pose the highest risk, macrocyclic chelates are of lowest risk, and the risk of linear ionic chelates falls between these two (37). Macrocyclic gadolinium contrast agents exhibit less demetallation, and ionic gadolinium contrast agents also tend to be more tightly bound (36).

A history of renal dysfunction or significant infirmity increases the risk of developing NSF $(29,36)$. Gadolinium contrast use is contraindicated in patients at high risk of NSF $(29,36)$. High-risk patients are those who have a kidney or liver transplant with a GFR of less than $60 \mathrm{~mL} / \mathrm{min} /$ $1.73 \mathrm{~m}^{2}$, a GFR of less than $30 \mathrm{~mL} / \mathrm{min} / 1.73 \mathrm{~m}^{2}$, or acute renal failure and who have one or more of the following comorbid conditions: major infection, vascular ischemia of extremities, vascular thrombosis, major surgery, major vascular procedure, or multiorgan system failure $(29,36)$.

Given the long biologic half-life of free gadolinium and the long retention of lanthanides in bone, it is important to consider the lifelong burden of gadolinium, especially in those have multiple contrast studies over their life-span (30).

Most, if not all, NSF cases after gadolinium contrast administration have significant renal dysfunction before administration $(36,37)$. The worse the renal function at the time of administration, the higher is the risk of NSF (36). The greater the total contrast dose, the greater are the risk and severity of NSF (36). Most patients who received gadolinium contrast agents, despite having significant renal dysfunction, do not get NSF (36).

NSF usually develops clinically within days to months after gadolinium exposure, although rare cases have been reported years later (36). Renal dysfunction causes a prolonged elimination half-life, and the retention of gadolinium increases the predisposition of $\mathrm{Gd}^{3+}$ to be displaced in the chelate by other metal cations such as iron $\left(\mathrm{Fe}^{3+}\right)$, copper $\left(\mathrm{Cu}^{2+}\right)$, zinc $\left(\mathrm{Zn}^{2+}\right)$, or calcium $\left(\mathrm{Ca}^{2+}\right)$ in a process called transmetallation (30). At the same time, several anions can compete for the $\mathrm{Gd}^{3+}$ ligand, including phosphate, carbonate, hydroxide, and citrate (30). The free gadolinium ion $\left(\mathrm{Gd}^{3+}\right)$ can then be deposited in skin and soft tissue to precipitate NSF (30). Procedural changes, improved contrast agents, and prescreening of high-risk patients have virtually eliminated this iatrogenic condition. In the absence of severe renal dysfunction, free gadolinium can manifest as gadolinium-induced plaques in the extremities. Transmetallation of the cation and exchange of the anion may cause spurious pseudohypocalcemia (30).

\section{Management of Adverse Reactions}

Given the absence of treatment for NSF, the remainder of the adverse reactions can and should be managed in the same way as outlined above for iodinated contrast agents based on the type of adverse reaction and symptoms (Fig. 4).

\section{Extravasation}

Fast mechanical injectors with large volumes for MRI contrast agents increase the risk of full or partial dose extravasation (29). Given the hypertonic nature of gadolinium contrast media, the effects and treatment are the same as those outlined above for iodinated CT contrast media. Indeed, the prevention strategies and risk factors are also similar.

\section{Interactions}

Specific interactions between gadolinium contrast agents and medications have not been widely evaluated or reported (26). The common properties of gadolinium contrast agents and iodinated contrast agents (iconicity, osmolality) present a similar interaction profile, although the lower doses of gadolinium truncate interaction risk. Like iodinated contrast agents, gadolinium contrast agents are not highly active pharmacologically (26). Prevention relies on obtaining a thorough patient history, being aware of drugs needing precautions, and not administering contrast through the same line as medications (26). Ionic gadolinium contrast agents have anticoagulant properties, and both ionic and nonionic can prolong coagulation time, possibly potentiating the effects of anticoagulant medications (e.g., heparin and warfarin), antiplatelet medications (e.g., aspirin and NSAIDs), and fibrinolytic medications (e.g., urokinase) (26).

Gadolinium contrast agents are not considered to pose a high risk for nephrotoxicity so do not substantially increase the bioavailability of renally eliminated medications; however, nephrotoxic medications such as NSAIDs and gentamicin can compound renal dysfunction to increase the risk of NSF (26). Adverse reactions to gadolinium contrast agents are more likely if the patient is taking immunomodulator medications (26). Allergic reactions or symptoms of a similar nature are more likely in patients taking $\beta$-blockers, interleukins, and interferons (26). Synergistic effects between gadolinium contrast media and calcium channel blockers and digoxin are possible (26). Higher-osmolality gadolinium contrast agents used 
for cerebral angiography lower the seizure threshold of antipsychotics, thioxanthenes, antidepressants, and analeptics (26).

From a nuclear medicine perspective, use of gadolinium contrast agents in the $72 \mathrm{~h}$ before ${ }^{67} \mathrm{Ga}$-citrate administration is known to alter biodistribution, as is evident in the more defined skeletal accumulation of the ${ }^{67} \mathrm{Ga}$. Gallium is an iron analog and can readily undergo transmetallation with gadolinium, and exchange between citrate and the chelate can also occur.

\section{CONCLUSION}

CT and MRI contrast agents are unique interventions that demand an expanded skill set and understanding of basic and applied pharmacology (1-5). Having insight into the complexities of both iodinated and gadolinium contrast agents enhances practice and patient safety. Specifically, a command of adverse reactions is a key skill required for capability in CT or MRI and ensures that nuclear medicine technologists meet the minimum capabilities for their scope of practice.

\section{DISCLOSURE}

No potential conflict of interest relevant to this article was reported.

\section{REFERENCES}

1. Currie GM. Pharmacology, part 1: introduction to pharmacology and pharmacodynamics. J Nucl Med Technol. 2018;46:81-86.

2. Currie GM. Pharmacology, part 2: introduction to pharmacokinetics. J Nucl Med Technol. 2018;46:221-230.

3. Currie GM. Pharmacology, part 3A: interventional medications in renal and biliary imaging. J Nucl Med Technol. 2018;46:326-334.

4. Currie GM. Pharmacology, part 3B: less commonly used interventional medications and adjunctive medications in general nuclear medicine. J Nucl Med Technol. 2019;47:3-12.

5. Currie GM. Pharmacology, part 4: nuclear cardiology. J Nucl Med Technol. February 15, 2019 [Epub ahead of print].

6. Bourin M, Jolliet P, Ballereau F. An overview of the clinical pharmacokinetics of X-ray contrast media. Clin Pharmacokinet. 1997;32:180-193.

7. Meurer K, Kelsch B, Hogstrom B. The pharmacokinetic profile, tolerability and safety of the iodinated, non-ionic, dimeric contrast medium Iosimenol 340 injection in healthy human subjects. Acta Radiol. 2015;56:581-586.

8. Geenen RW, Kingma HJ, van der Molen AJ. Contrast-induced nephropathy: pharmacology, pathophysiology and prevention. Insights Imaging. 2013;4:811-820.

9. Block JH, Beale JM. Wilson and Gisvold's Textbook of Organic Medicinal and Pharmaceutical Chemistry. 12th ed. Philadelphia, PA: Lippincott Williams and Wilkins; 2011.

10. Pasternak JJ, Williamson EE. Clinical pharmacology, use, and adverse reactions of iodinated contrast agents: a primer for non-radiologists. Mayo Clin Proc. 2012;87:390-402.

11. Namasivayam S, Kalra MK, Torres WE, Small WC. Adverse reactions to intravenous iodinated contrast media: a primer for radiologists. Emerg Radiol. 2006;12:210-215.

12. Costa N. Understanding contrast media. J Infus Nurs. 2004;27:302-312.
13. Iyer RS, Schopp JG, Swanson JO, Thapa MM, Phillips GS. Safety essentials: acute reactions to iodinated contrast media. Can Assoc Radiol J. 2013;64:193-199.

14. Bae KT. Intravenous contrast medium administration and scan timing at CT: considerations and approaches. Radiology. 2010;256:32-61.

15. Seibert JA, Boone JM. X-ray imaging physics for nuclear medicine technologists. Part 2: x-ray interactions and image formation. J Nucl Med Technol. 2005;33:3-18.

16. Sweetman SC, ed. Martindale: The Complete Drug Reference. 26th ed. Chicago, IL: Pharmaceutical Press; 2009.

17. Morzycki A, Bhatia A, Murphy KJ. Adverse reactions to contrast material: a Canadian update. Can Assoc Radiol J. 2017;68:187-193.

18. Namasivayam S, Kalra MK, Torres WE, Small WC. Adverse reactions to intravenous iodinated contrast media: an update. Curr Probl Diagn Radiol. 2006;35:164-169.

19. Bryant B, Knights K, Salerno E. Pharmacology for Health Professionals. 2nd ed. Sydney, Australia: Mosby Elsevier; 2007.

20. Rang H, Dale M, Ritter J, Flower R. Rang and Dale's Pharmacology. 6th ed. London, U.K.: Churchill Livingston; 2008.

21. Cohan RH, Ellis JH, Garner WL. Extravasation of radiographic contrast material: recognition, prevention, and treatment. Radiology. 1996;200:593-604.

22. Ayre-Smith G. Tissue necrosis following extravasation of contrast material. J Can Assoc Radiol. 1982;33:104.

23. Jacobs JE, Birnbaum BA, Langlotz CP. Contrast media reactions and extravasation: relationship to intravenous injection rates. Radiology. 1998;209:411416.

24. Federle MP, Chang PJ, Confer S, Ozgun B. Frequency and effects of extravasation of ionic and nonionic CT contrast media during rapid bolus injection. Radiology. 1998;206:637-640.

25. Wang CL, Cohan RH, Ellis JH, Adusumilli S, Dunnick NR. Frequency, management, and outcome of extravasation of nonionic iodinated contrast medium in 69,657 intravenous injections. Radiology. 2007;243:80-87.

26. Morcos SK, Thomsen HS, Exley CM. Contrast media: interactions with other drugs and clinical tests. Eur Radiol. 2005;15:1463-1468.

27. Bellin MF, Van Der Molen AJ. Extracellular gadolinium-based contrast media: an overview. Eur J Radiol. 2008;66:160-167.

28. Terreno E, Aime S. MRI contrast agents in pharmacological research. Front Pharmacol. 2015;6:290.

29. Lin SP, Brown JJ. MR contrast agents: physical and pharmacologic basics. J Magn Reson Imaging. 2007;25:884-899.

30. Abraham JL, Thakral C. Tissue distribution and kinetics of gadolinium and nephrogenic systemic fibrosis. Eur J Radiol. 2008;66:200-207.

31. Behzadi AH, Zhao Y, Farooq Z, Prince MR. Immediate allergic reactions to gadolinium-based contrast agents: a systemic review and meta-analysis. Radiology. 2018;286:471-482.

32. Bruder O, Schneider S, Nothnagel D, et al. Acute adverse reactions to gadoliniumbased contrast agents in CMR. JACC Cardiovasc Imaging. 2011;4:1171-1176.

33. Aran S, Shaqdan KW, Abujudeh HH. Adverse allergic reactions to linear ionic gadolinium-based contrast agents: experience with 194,400 injections. Clin Radiol. 2015;70:466-475.

34. Jung JW, Kang HR, Kim MH, et al. Immediate hypersensitivity reaction to gadolinium-based MR contrast media. Radiology. 2012;264:414-422.

35. Radbruch A, Weberling LD, Kieslich PJ, et al. Gadolinium retention in the dentate nucleus and globus pallidus is dependent on the class of contrast agent. Radiology. 2015;275:783-791.

36. Kanal E. Gadolinium based contrast agents (GBCA): safety overview after 3 decades of clinical experience. Magn Reson Imaging. 2016;34:1341-1345.

37. Khawaja AZ, Cassidy DB, Shakarchi JA, McGrogan DG, Inston NG, Jones RG. Revisiting the risks of MRI with gadolinium based contrast agents: review of literature and guidelines. Insights Imaging. 2015;6:553-558. 